\title{
A Técnica de Rorschach e os Critérios da CID-10 para o Diagnóstico da Esquizofrenia
}

\author{
The Rorschach Technique and the ICD-10 Criteria for Schizophrenia Diagnosis
}

\author{
Ana Cristina Resende* a \& Irani Iracema de Lima Argimon ${ }^{b}$ \\ ${ }^{a}$ Pontificia Universidade Católica de Goiás, Goiânia, Brasil \\ \& ${ }^{b}$ Pontifícia Universidade Católica do Rio Grande do Sul, Porto Alegre, Brasil
}

\begin{abstract}
Resumo
Trata de um estudo de correlação entre as variáveis da técnica de Rorschach (Sistema Klopfer) e os grupos de sinais e sintomas da CID-10 para a esquizofrenia. Participaram desse estudo 80 pacientes com o diagnóstico de esquizofrenia pela CID-10, com recidiva da doença, internados em hospitais psiquiátricos de Goiânia. Observou-se que, dentre os 8 grupos de sinais e sintomas da CID-10 para esquizofrenia, 7 grupos tiveram correlações com variáveis distintas do Rorschach. Tais dados sugerem que, embora exista uma relação entre as variáveis dos dois instrumentos, estas variáveis não se sobrepõem substancialmente. Entende-se que são importantes as pesquisas que indiquem padrões de inter-relação entre diferentes modelos de avaliação de distúrbios psíquicos, a fim de que se possam apontar pontos de convergência e divergência entre os mesmos, avançando desse modo em questões conceituais.

Palavras-chave: Esquizofrenia, técnica de Rorschach, CID-10.
\end{abstract}

\begin{abstract}
This is a correlational study between the variables of the Rorschach technique (Klopfer System) and groups of signals and symptoms of ICD-10 for schizophrenia. Eighty patients diagnosed with schizophrenia by the ICD-10, with disease recidivation, who were hospitalized in a psychiatric service in the city of Goiânia participated in the study. We observed that among the 8 groups of signals and symptoms of ICD10 for schizophrenia, 7 groups had correlations with distinct variable of Rorschach. These data suggest that while there is a relationship between the variables of the two instruments, such variables do not overlap substantially. It is understood that researches are important to indicate patterns of interrelationship between different models for evaluation of mental disorders so that they can point out similarities and differences between them, thereby advancing the conceptual issues.
\end{abstract}

Keywords: Schizophrenia, Rorschach technique, ICD-10.

$\mathrm{Na}$ introdução deste artigo serão expostos os critérios diagnósticos da CID-10 (Organização Mundial de Saúde [OMS], 1993) para a esquizofrenia, adotados na pesquisa, como também serão feitas algumas explanações a respeito da técnica de Rorschach e essa psicopatologia, uma vez que a relação entre esses dois instrumentos é o foco deste estudo. Tanto um quanto o outro instrumento, isoladamente, têm demonstrado que seus achados são úteis na avaliação clínica de transtornos esquizofrênicos (Exner, 2003; Jakobsen et al., 2005; Kleiger, 1999; Weiner, 1966). No entanto, quando se trata de relacionar o Rorschach com um outro instrumento de classificação diagóstica nota-se que a correlação entre eles tende a ser baixa

\footnotetext{
*Agradecimento especial à Coordenação de Aperfeiçoamento de Pessoal de Nível Superior (CAPES) por viabilizar financeiramente este estudo.

Endereço para correspondência: Departamento de Psicologia - Área IV, Pontifícia Universidade Católica de Goiás, Av. Universitária 1.440, Setor Universitário, Goiânia, GO, Brasil 74605-010. Tel.: (62) 3946-1097 ou (62) 9137-0535. E-mail: anacristinaresende@hotmail.com e argimoni@pucrs.br
}

(Wood \& Lilienfeld,1999; Wood, Lilienfeld, Garb, \& Nezworski, 2000) e quando se trata de verificar essa relação entre o Rorschach e os critérios diagnósticos da CID-10 para esquizofrenia nenhum estudo foi encontrado nas bases de dados que se referem a estudos em ciências humanas e da saúde.

No Brasil, poucos estudos têm averiguado pontos de convergência e divergência entre diferentes modelos de avaliação de psicopatologias e seus respectivos instrumentos. Os desafios na área da avaliação de psicopatologia não se resumem a questões técnicas de construção e validação de instrumentos. Constata-se a necessidade de pesquisas que indiquem padrões de inter-relação entre os diversos modelos de avaliação de distúrbios psíquicos existentes, a fim de que se possam apontar pontos de convergência e divergência entre os mesmos, avançando desse modo em questões conceituais. Segundo Pasquali (1999), mesmo que as investigações tenham objetivos modestos, parciais ou focalizem apenas uma pequena parte das necessidades da área, tais pesquisas ainda são relevantes e necessárias. 


\section{Critérios Diagnósticos de Esquizofrenia}

Adotados na Pesquisa

O presente estudo ater-se-á aos critérios, às descrições conceituais e às diretrizes diagnósticas da CID-10 (OMS, 1993) para esquizofrenia. Independentemente de qualquer apriorismo teórico, os profissionais responsáveis por esse manual tentaram criar uma nomenclatura única para pesquisadores e clínicos mediante a observação direta do fenômeno patológico.

Os sintomas característicos da doença, definidos pela CID-10, se manifestam no paciente, com certa frequência, em conjunto, e se distribuem em oito grupos. Os quatro primeiros se caracterizam por delírios e alucinações tais como:

1. Eco, inserção, roubo ou irradiação de pensamento.

2. Delírios de controle, influência ou passividade e percepção delirante.

3. Vozes alucinatórias comentando o comportamento do paciente ou discutindo entre elas ou outros tipos de vozes alucinatórias vindas de alguma parte do corpo.

4. Delírios persistentes de outros tipos, culturalmente inapropriados e completamente impossíveis como poderes e capacidades sobre-humanas.

Os quatro últimos grupos de sintomas caracterizam por:

5. Alucinações persistentes em qualquer modalidade, quando acompanhadas por delírios "superficiais" ou parciais, sem claro conteúdo afetivo, ou por idéias sobrevaloradas persistentes ou quando ocorrem todos os dias durante semanas ou meses continuadamente.

6. Neologismos, paradas ou interpolação no curso do pensamento, resultando em incoerência ou fala irrelevante.

7. Comportamento catatônico, tal como excitação, postura inadequada ou flexibilidade cérea, negativismo, mutismo e estupor.

8. Sintomas "negativos" como apatia marcante, pobreza de discurso e embotamento ou incongruência de respostas emocionais, usualmente resultando em retraimento e diminuição do desempenho social (deve estar claro que estas não são devidas a depressão ou medicação neuroléptica).

Diagnostica-se a esquizofrenia (F20) se pelo menos um dos sintomas dos quatro primeiros grupos, ou, no mínimo, dois dos sintomas dos quatro últimos grupos estiverem evidentes na maior parte do tempo, por um período de um mês ou mais. São, por outro lado, critérios excludentes do diagnóstico de esquizofrenia: a presença de uma doença cerebral clara; evidência de estados de intoxicações ou de abstinência de drogas; a existência incontestável de sintomas de depressão ou mania antecedendo outros sintomas que caracterizam esta doença.

\section{Variáveis do Rorschach para a Esquizofrenia}

O Rorschach é uma técnica de avaliação de personalidade construída por Hermann Rorschach, em 1921, na Suíça. Esse instrumento é reconhecido mundialmente por sua eficácia na avaliação do funcionamento psíquico de crianças, jovens e adultos, quer sejam considerados normais ou diagnosticados com alguma psicopatologia.

A técnica de Rorschach, que não foi construída especificamente para aferição diagnóstica de acordo com os critérios da CID-10 (OMS, 1993), tem se mostrado eficiente para a descrição psicológica da esquizofrenia segundo vários estudiosos que fizerem o uso do instrumento para o estudo dessa psicopatologia. Dentre esses estudiosos destaca-se o próprio criador do instrumento, Hermann Rorschach (Rorschach, 1921/1978) além de uma série de outros autores: Adrados (1991, 2004); Amparo (2002); Beizmann (1968); Blatt e Wild (1976); Bodoin e Pikunas (1983); Bohm (1979); Colombo et al. (1993); Exner e Sendín (1999); Ilonen et al. (2004); Kaser-Boyd (2006); Kleiger (1999); Klinger e Roth (1965); Klopfer e Kelly (1952); Koide, Chien, Iizuka e Morita (2002); Palem (1969, citado por Chabert, 1993); Passalacqua e Gravenhorst (2005); Portuondo (1972); Resende (2001); Santos (1996); Shereshevski-Shere, Lasser e Gottsfeld (1953); Silva (1987); Sousa (1982); Traubenberg (1998) e Vaz (1997).

Destacam-se na identificação de variáveis indicadoras de esquizofrenia no Rorschach os estudos dos investigadores e autores: Hermann Rorschach (1921/1978), pelo mérito de iniciar o seu método na investigação de pacientes esquizofrênicos; Klopfer e Kelly (1952) e Vaz (2006) por esse estudo propor a análise da técnica de Rorschach mediante o sistema de classificação e interpretação desses autores; Bohm (1979) e Portuondo (1972), considerados autores clássicos na investigação da esquizofrenia por meio do Rorschach; Sousa (1982), Adrados (1991, 2004), Chabert (1993) e Exner e Sendín (1999) por serem referências mais recentes e por descreverem as variáveis do Rorschach mais comuns em pacientes diagnosticados com o transtorno de esquizofrenia de uma forma geral, sem privilegiar a distinção entre as várias classificações dessa doença (paranóide, catatônica, hebefrênica, etc.) ou o estudo de algumas variáveis específicas no Rorschach.

De acordo com um levantamento de variáveis do Rorschach para a esquizofrenia, realizado entre os autores citados anteriormente (ver Tabela 1), observou-se que a presença das variáveis Contaminação e Confabulação, além da alta frequência da Forma de Má Qualidade (F-) e baixa frequência das Respostas Populares (Pop), são as mais frequentemente citadas como características da esquizofrenia, embora não sejam exclusivas desse transtorno. Essa constatação remete à questão da eficiência do Rorschach na descrição da esquizofrenia, pois os vários estudos confirmam sua validade e fidedignidade para o estudo dessa psicopatologia. Todas as variáveis frequentemente encontradas revelam alterações graves na percepção do estímulo da técnica e, consequentemente, da realidade. Tais prejuízos se assemelham aos observados pelos sintomas de delírios e alucinações nos esquizofrênicos. 
Tabela 1

Variáveis Indicadoras de Esquizofrenia no Rorschach por Diferentes Autores/Pesquisadores

\begin{tabular}{|c|c|c|c|c|c|c|c|c|c|}
\hline $\begin{array}{l}\text { Autores } \\
\text { Variáveis do } \\
\text { Rorschach }\end{array}$ & r. & 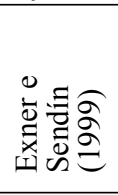 & 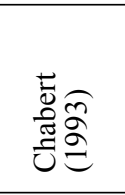 & 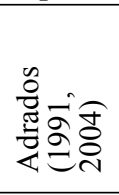 & 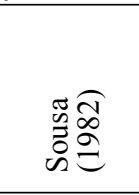 & छ⿳亠口冋亍 & 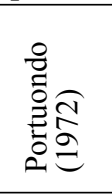 & 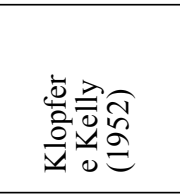 & 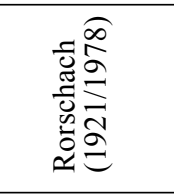 \\
\hline Contaminação & $*$ & $*$ & & * & * & * & * & * & * \\
\hline Confabulação & & $*$ & $*$ & $*$ & $*$ & $*$ & $*$ & $*$ & $*$ \\
\hline Resposta Bizarra & & & $*$ & & & & $*$ & & \\
\hline $\mathrm{G}^{\uparrow}\left(\mathrm{G}->\mathrm{G}^{+}\right)$ & & & * & * & * & * & * & * & \\
\hline $\mathrm{Dd} \uparrow(\mathrm{Dd}->\mathrm{Dd}+)$ & & & $*$ & * & * & * & * & $*$ & \\
\hline$S \uparrow$ & & & $*$ & * & * & & & & \\
\hline Sucessão confusa & & & & * & & * & & * & * \\
\hline$F \% \downarrow$ & * & & * & & & & & & \\
\hline$F+\% \downarrow$ ou $F-\% \uparrow$ & * & * & * & * & * & * & * & * & * \\
\hline $\mathrm{FC}=0$ & $*$ & & & $*$ & & & $*$ & & \\
\hline $\mathrm{FC}<\mathrm{CF}+\mathrm{C}$ & & & & & $*$ & & & $*$ & \\
\hline$\sum \mathrm{C} \downarrow$ & & & & & & $*$ & & & \\
\hline C e C' deterioradas & & & & & & & * & & \\
\hline $\mathrm{Cn}$ & * & & * & * & * & * & * & $*$ & \\
\hline Clob puro & & & & & & & * & & \\
\hline$M=0$ & $*$ & & $*$ & & & & $*$ & & \\
\hline$M \downarrow$ & & & & * & * & & & *paranóia $\uparrow$ & *paranóia $\uparrow$ \\
\hline$M-$ & $*$ & $*$ & & * & * & & & & \\
\hline$R \downarrow$ & * & * & * & & & & & & \\
\hline$P \downarrow$ & * & * & * & * & * & * & * & * & * \\
\hline $\mathrm{C}$ & $*$ & & & & & & & & \\
\hline$\sum \mathrm{H} \downarrow$ ou $=0$ & & $*$ & & & & & & & \\
\hline$H d>H$ & * & & * & $*$ & & * & & & \\
\hline $\mathrm{A} \% \uparrow$ & & & & * & & & $*$ & $*$ & \\
\hline At $\uparrow$ & & & * & * & & * & * & & \\
\hline Auto-referência & * & & * & * & * & * & * & * & * \\
\hline Crítica & * & & & & & * & & & \\
\hline Olhar o verso & * & & & & & & & & \\
\hline Olhar anotações & * & & & & & & & & \\
\hline Extratensivo $^{1}$ & $*$ & & $*$ & $*$ & $*$ & $*$ & & $*$ & \\
\hline Introversivo & & * & & & & & & & \\
\hline Coartado & & & & * & & * & * & & \\
\hline Número & & & & * & * & * & & & \\
\hline Letras e Geometria & & & & & & * & & & \\
\hline Objeto $\uparrow$ & & & & & & & * & & \\
\hline Original $\uparrow(\mathrm{O}->\mathrm{O}+)$ & & & & * & * & * & * & $*$ & * \\
\hline Fragm+doença+morte & & & * & & & * & * & & \\
\hline Abstração & & * & * & * & * & * & * & * & * \\
\hline Perseveração & & & $*$ & $*$ & $*$ & $*$ & $*$ & $*$ & $*$ \\
\hline Neologismo & & & & $*$ & $*$ & & & & \\
\hline Projeção de Cor & & & * & $*$ & & & & & \\
\hline Descrição do cartão & & & & $*$ & & $*$ & & * & * \\
\hline Choque VII e IX & & & & $*$ & & & & & \\
\hline Tempo Reação & & & elevado & & instável & rápido & rápido & & \\
\hline Rejeição & & $*$ & $*$ & $*$ & & $*$ & & $*$ & $*$ \\
\hline Fracasso em pr. fácil (V) & & & & * & & $*$ & & & \\
\hline Fusão Figura-fundo & & & & & & * & * & & \\
\hline $\mathrm{XA} \downarrow$ & & $*$ & & & & & & & \\
\hline $\mathrm{Ke} \mathrm{c}$ & & & & & & & & * & \\
\hline Posição & & & & * & $*$ & $*$ & $*$ & * & * \\
\hline
\end{tabular}

Notas. Fontes consultadas: I. Adrados (1991, pp. 150-155 e 2004, pp. 24-48), E. Bohm (1979, p. 336-338), C. Chabert (1993, pp. 115-116 e 135-145), J. E. Exner e C. Sendín (1999, p. 50), B. Klopfer e D. Kelly (1952, pp. 252-270), A. J. Portuondo (1972, pp. 3941), H. Rorschach (1921/1978, pp. 292-294), C. C. Sousa (1982, p. 124), C. E. Vaz (1997, pp. 121-122 e 2006, p. 100).

* Indica a presença da variável do Rorschach. $\uparrow$ Índice considerado elevado e $\downarrow$ Índice considerado baixo. 
A Contaminação é o sinal mais grave de dissociação do pensamento na técnica de Rorschach, pois a pessoa faz uma síntese absurda de elementos percebidos na mancha, violando o princípio de realidade, que se fossem percebidos separadamente, e não fundidos, poderiam ser considerados plausíveis. A Confabulação se traduz como uma fuga da realidade por meio do uso comprometedor da fantasia e revela grave perturbação do pensamento causada pela deterioração da esfera conceitual e pela liberação do emotivo e dinâmico, os quais passam a modelar a realidade. Neste caso, a pessoa inicia o processo de verbalização de sua resposta ao teste num detalhe e segue como que montando uma estória, usando a imaginação fantasiosa em torno do que segue falando (Vaz, 1997).

$\mathrm{O} F$ - indica prejuízo ou comprometimento do funcionamento do pensamento lógico quanto à clareza, à precisão, à coerência e à organização. Está relacionado a uma maior probabilidade de a pessoa formar impressões errôneas de si mesmo, do outro e da realidade. Vaz (1997) descreve que no teste a pessoa verbaliza um conteúdo cuja forma, muitas vezes confusa ou vaga, não se adequada ao contorno da mancha ou, por outro lado, o conteúdo verbalizado é combinado com algum fenômeno especial que indica deslizes cognitivos (por exemplo, a própria confabulação e contaminação citadas anteriormente).

Quanto à baixa frequência de Respostas Populares, indica pouco senso comum e limitações que a pessoa tem para perceber a realidade objetiva e precisa, em consonância com o grupo a que pertence. Segundo Vaz (1997), o critério para que uma reposta fosse considerada popular foi de 1/6 dos sujeitos avaliados verbalizarem um mesmo conteúdo numa mesma localização da mancha. Assim, quando qualquer pessoa avaliada emitir qualquer um dos 15 conteúdos que alcançaram este critério de freqüência, sua resposta é considerada popular.

Dessa forma, considerando a validade da técnica de Rorschach para o estudo da esquizofrenia e a CID-10 como um instrumento de classificação oficial dos transtornos mentais e do comportamento, destinado ao uso clínico, educacional, assistencial em geral e pesquisa, de reconhecido valor científico pela OMS, o presente estudo teve como objetivo investigar empiricamente as possíveis relações entre as variáveis desses dois instrumentos no que tange ao diagnóstico da esquizofrenia. As duas questões principais seriam investigar o quanto e de que maneira esses dois instrumentos, que podem ajuizar a respeito de um diagnóstico de esquizofrenia em avaliações clínicas, estão correlacionados. A expectativa geral é que haja uma correlação estatisticamente significativa entre algumas variáveis e ausência de correlação entre outras.

\section{Método}

\section{Participantes}

Participaram do estudo 80 pessoas com o diagnóstico de esquizofrenia (F20), sendo quarenta homens e qua- renta mulheres, com recidiva da doença, provenientes de três hospitais psiquiátricos de Goiânia, que faziam uso de medicação para tratamento dessa enfermidade. Os médicos faziam uma primeira seleção entre os seus pacientes que poderiam participar do estudo considerando especialmente os critérios de exclusão descritos logo abaixo e, posteriormente, a pesquisadora responsável verificava de forma minuciosa, mediante informações descritas nos prontuários, se cada um dos pacientes atendiam aos critérios de inclusão e não se encaixavam nos critérios de exclusão.

Os participantes deveriam atender aos seguintes critérios de inclusão: recidiva da doença; apresentar registro em prontuário de duas ou mais internações na instituição, como também ter o seu diagnóstico confirmado por no mínimo dois psiquiatras, ter o registro de características socioeconômicas, sintomas clínicos, se havia alguma comorbidade, antecedentes familiares, início da doença; ter entre 20 e 55 anos; concordar em participar do estudo e ter o seu consentimento livre e esclarecido assinado por um de seus responsáveis.

Foram excluídos aqueles pacientes em que houve uma manifestação de episódio maníaco (F30), ou de episódio depressivo (F32), anteriores aos sintomas característicos de esquizofrenia, bem como aqueles pacientes em que se detectou a presença de doença cerebral orgânica, estado de intoxicação, dependência, abstinência de álcool ou drogas. Estes critérios de exclusão também foram identificados nos prontuários dos pacientes, pois muitos deles tinham o histórico de sucessivas internações na própria instituição. Além disso, contou-se com as observações que os psiquiatras faziam no questionário referente a cada um de seus clientes, participantes deste estudo, após a entrevista psiquiátrica.

Setenta por cento desses pacientes $(n=56)$ estavam sendo medicados com antipsicóticos típicos incisivos, do tipo Haloperidol. Os pacientes permaneciam internados nesses hospitais por um período médio de trinta dias e muitos deles ainda estavam em crise. O nível socioeconômico dessas pessoas era relativamente baixo. Entre os homens as profissões que mais predominaram foram a de trabalhador rural e servente $(18 \%$ da amostra, $n=14$ ); entre as mulheres, a maioria era do lar ou doméstica $(35 \%, n=28)$. Grande parte $(85 \%, n=68)$ estava sob tratamento pelo Sistema Único de Saúde (SUS). As idades dos participantes variaram entre $20 \mathrm{e}$ 55 anos $(M=38$ anos, $D P=9,5)$, predominava o estado civil solteiro $(66,3 \%, n=53)$ e a escolaridade média foi de 6,5 anos de estudo $(D P=3,1)$. Na Tabela 2 observase maiores informações sobre os dados sociodemográficos dos sujeitos.

O tamanho da amostra $(n=80)$ foi determinado em função da prevalência da enfermidade na população mundial, ou seja, $1 \%$, considerando que, em média, $50 \%$ dos pacientes possuem comorbidade com abuso de substâncias alcoólicas ou outras drogas (Volkow, 2009) e, portanto, não poderiam participar do estudo. Para calcu- 
Tabela 2

Estatística Descritiva das Variáveis Sociodemográficas dos Sujeitos do Estudo

\begin{tabular}{|c|c|c|c|c|c|}
\hline & Variáveis & $f$ & $\%$ & $M$ & $D P$ \\
\hline \multirow{5}{*}{ Idade } & $20-29$ & 16 & 22,5 & \multirow{4}{*}{38} & \multirow{4}{*}{9,5} \\
\hline & $30-39$ & 30 & 37,5 & & \\
\hline & $40-49$ & 20 & 25,0 & & \\
\hline & $50-55$ & 12 & 15,0 & & \\
\hline & Total & 80 & 100,0 & & \\
\hline \multirow{4}{*}{ Anos de Estudo } & $1-5$ & 32 & 40,0 & \multirow{3}{*}{6,5} & \multirow{3}{*}{3,1} \\
\hline & $6-10$ & 38 & 47,5 & & \\
\hline & $11-17$ & 10 & 12,5 & & \\
\hline & Total & 80 & 100,0 & & \\
\hline \multirow{11}{*}{ Profissão } & Carpinteiro & 02 & 2,5 & \multirow[t]{10}{*}{-} & \multirow[t]{10}{*}{-} \\
\hline & Do lar & 18 & 22,5 & & \\
\hline & Doméstica & 10 & 12,5 & & \\
\hline & Estudantes & 05 & 6,3 & & \\
\hline & Frentista & 02 & 2,5 & & \\
\hline & Pedreiro & 04 & 5,0 & & \\
\hline & Servente & 06 & 7,5 & & \\
\hline & Trab. Rural & 09 & 11,3 & & \\
\hline & Vendedor & 03 & 3,7 & & \\
\hline & Outras & 21 & 26,2 & & \\
\hline & Total & 80 & 100,0 & & \\
\hline \multirow{5}{*}{ Estado Civil } & Casado & 16 & 20,0 & \multirow{4}{*}{-} & \multirow{4}{*}{-} \\
\hline & Divorciado & 3 & 3,7 & & \\
\hline & Separado & 8 & 10,0 & & \\
\hline & Solteiro & 53 & 66,3 & & \\
\hline & Total & 80 & 100,0 & & \\
\hline \multirow{3}{*}{ Plano de Saúde } & SUS & 68 & 85,0 & \multirow{2}{*}{ - } & \multirow{2}{*}{$-{ }_{-}^{*}$} \\
\hline & Particular & 12 & 15,0 & & \\
\hline & Total & 80 & 100,0 & & \\
\hline
\end{tabular}

lar o tamanho mínimo da amostra foi aplicada a seguinte fórmula sugerida por Barbetta (2004): $n_{0}=1 / e^{2}$ and $n$ $=N . n_{\delta} / N+n_{0}$. O $N$ consiste no tamanho da população (aproximadamente 6.000 pacientes esquizofrênico sem comorbidade com abuso de substâncias), o $n$ constitui o tamanho da amostra, o $n_{0}$ equivale a uma primeira aproximação para o tamanho da amostra e o $e$ trata-se do erro amostral tolerável (determinado pelo pesquisador, 11\%).

\section{Instrumentos para Coleta de Dados}

Entrevista Psiquiátrica contendo os Oito Critérios da CID-10 compatíveis com o diagnóstico de esquizofrenia. Tratava-se de uma entrevista regular em que o psiquiatra conversava com o paciente para ver como andava o tratamento e a remissão dos sintomas psicóticos. Adicionalmente, foi solicitado aos psiquiatras que, durante esta entrevista regular, verificassem a presença ou não de cada um dos oito grupos de sinais e sintomas da CID-10 para o diagnóstico da esquizofrenia, que eram anotados em um questionário objetivo, criado para facilitar o levantamento dos critérios. O psiquiatra deveria marcar com um $\mathrm{X}$ aqueles grupos de sintomas que estavam presentes em cada um de seus pacientes. No questionário também havia espaço para o psiquiatra fazer as observações que achasse necessária. Esta entrevista tinha o objetivo de especificar quais seriam os critérios do diagnóstico de esquizofrenia, dentre os oito elencados na literatura, que levaram o psiquiatra a definir o diagnóstico do paciente. Esta etapa foi imprescindível para que se fizesse a correlação entre as variáveis do Rorschach e os grupos de sintomas da CID-10, uma vez que os médicos não têm a prática de apontar nos prontuários quais dos oito grupos de sintomas estavam presentes no momento do diagnóstico diferencial.

Entre os vários ensaios atuais que sustentam confiabilidade entre os profissionais da área da saúde e consti- 
tuem uma das ferramentas provisórias e pragmáticas mais conhecidas e usadas na clínica e na pesquisa destaca-se a CID-10 (OMS, 1993). O presente estudo ateve-se aos critérios, às descrições conceituais e às diretrizes diagnósticas da CID-10 (OMS, 1993) para esquizofrenia, por se tratar de uma classificação oficial dos transtornos mentais e do comportamento, destinada ao uso clínico, educacional, assistencial em geral, bem como ao desenvolvimento de pesquisa. Além desse motivo, o estudo foi executado na cidade de Goiânia, onde os médicos psiquiatras, que de uma forma ou de outra colaboraram com a pesquisa, adotam sistematicamente a CID-10.

Técnica de Rorschach. Consiste em dez cartões contendo manchas de tinta feitas pelo seu criador. Esses estímulos não estruturados mobilizam processos de percepção, associação, projeção e de expressão verbal à medida que o examinando verbaliza o que percebe. Sobre as verbalizações emitidas, transformadas em variáveis de dados quantitativos e qualitativos, elaboraram-se sistemas de escores que permitem estudos de fidedignidade e validade, além de estudos normativos disponíveis no Brasil (Nascimento, 2007; Pasian, 2000; Vaz, 1997, 2006). Foi adotado o Sistema de Classificação de Klopfer, adaptado e validado por $\operatorname{Vaz}(1997,2006)$, com a complementação de algumas variáveis, ou categorias de codificação do Sistema Compreensivo ([SC], Exner, 2003), especificamente os Conteúdos Mórbidos (MOR), as Abstrações (AB), os Movimentos Humanos sem qualidade formal ( $M$ none), as Respostas Desviantes de nível $2(D R 2)$ e a variável Cor-Sombreado (Cor-Somb). É importante destacar que essas variáveis do $\mathrm{SC}$ consistem em dados qualitativos que podem ser levantados nas verbalizações dos examinandos, durante a aplicação da técnica, sem qualquer incompatibilidade entre as duas formas de correção do Rorschach.

Assume-se que nem todos os critérios da CID-10 (OMS, 1993) podem correlacionar-se com variáveis do Rorschach, tais como sensações específicas, durações dos sintomas e outros aspectos que são mais bem identificados mediante a observação do comportamento ou fala do paciente.

Prontuários dos Pacientes. Usados para coletar dados complementares que descrevem os participantes do estudo: informações gerais tais como profissão, idade, escolaridade, sintomas pregressos do tratamento, diagnósticos e medicação utilizada antes da avaliação por meio da técnica de Rorschach.

\section{Procedimentos para Coleta de Dados}

A diretoria de três hospitais psiquiátricos foi contatada para a obtenção do consentimento para realização do estudo em cada uma dessas instituições. Após a autorização do diretor de cada hospital, fez-se a triagem dos participantes com os médicos psiquiatras, considerando os critérios de inclusão e exclusão da amostra, que respondiam a um questionário especificando quais os critérios diagnósticos da CID-10 para esquizofrenia que cada paciente apresentava. Antes da administração da técnica de Rorschach, a primeira autora desse artigo, que fez todas as aplicações desta técnica, tomava nota de algumas informações no prontuário do paciente e conferia se realmente o paciente não tinha histórico de abuso de substâncias ou de qualquer outro critério de exclusão da amostra. Ainda previamente à testagem, foi mantido um contato com cada paciente a fim de convidá-lo e motivá-lo a participar da pesquisa, e obter o seu consentimento livre e esclarecido e de seu responsável.

A administração do Rorschach, em $75 \%$ dos casos $(n=60)$, foi realizada imediatamente após o psiquiatra do paciente responder ao questionário contendo os critérios da CID-10 para esquizofrenia. Os demais casos foram avaliados no período máximo de uma semana após o levantamento dos critérios da CID-10. Os testes não concluídos após esse intervalo foram descartados. Ao total houve uma perda de quatorze protocolos por esse motivo.

\section{Procedimentos para Análise de Dados}

Todos os protocolos foram codificados pela primeira autora deste trabalho. Outra psicóloga, que desconhecia os objetivos deste estudo, treinada na correção da técnica de Rorschach, tanto no Sistema Klopfer (Vaz, 1997) quanto no Sistema Compreensivo (Exner, 2003), codificou $25 \%$ dos protocolos, selecionados aleatoriamente, para que o estudo de concordância entre dois juízes (primeira autoras e a psicóloga cega aos objetivos do estudo) por meio do coeficiente Kappa fosse realizado. O passo seguinte consistiu em resolver as discordâncias entre os juízes por meio de uma discussão entre ambos para se decidir pela melhor codificação. Nos casos em que não foi possível um consenso entre os dois juízes, um terceiro juiz, que também desconhecia os objetivos do estudo, julgava a melhor codificação.

Para a verificação das possíveis correlações entre as variáveis dos instrumentos administrados foi realizada a análise de correlação pelo coeficiente de Kendall $(t-b)$, que é indicado no caso de variáveis dicotômicas, como é o caso das variáveis da CID-10, que constituem oito grupos de sinais e sintomas do transtorno esquizofrênico em que cada um desses grupos pode estar presente ou ausente em cada paciente. O nível de significância utilizado foi de no mínimo $5 \%(p<0,05)$.

No que se refere aos estudos normativos utilizados para classificar se as variáveis consideradas no estudo estavam aumentadas ou diminuídas, contou-se com as normas descritas em Vaz (1997 e 2006) para comparar as variáveis do Sistem Klopfer e as normas estabelecidas por Nascimento (2007) para as variáveis do Sistema Compreensivo.

\section{Resultados e Discussão}

A medida Kappa de concordância entre os juízes ( $n=$ 20 protocolos, respostas $=269$ ) indica que houve uma concordância alta entre os codificadores do teste e que a correção da técnica de Rorschach para esse estudo é considerada confiável. Os valores Kappa foram 0,86 para as 
categorias de Determinantes e Fenômenos Especiais, 0,89 para Conteúdos, 0,92 para Respostas Populares e 0,94 para Localizações (sendo $p<0,001$ para todas as categorias).

Os resultados das correlações entre os critérios da CID10 e variáveis do Rorschach para a esquizofrenia, por meio do coeficiente de Kendall $(t-b)$, são apresentados na Tabela 3. Para a construção dessa tabela fez-se todas as possíveis correlações entre as variáveis dos dois instrumentos.

De acordo com a Tabela 3, a variável Perseveração no Rorschach está presente de modo significativo em pacientes que apresentam os critérios 1 da CID-10 para esquizofrenia - eco do pensamento, inserção, roubo ou irradiação do pensamento. Entende-se que o eco do pensamento - uma voz sussurrada ou intoleravelmente forte que repete o pensamento do paciente pouco depois de tê-lo pensado - esteja relacionado à Perseveração, fenômeno indicador de compulsão à repetição de um mesmo conteúdo no Rorschach típicos de pessoas com distúrbios cognitivos e neurológicos graves (Exner, 2003; Vaz, 1997).

Tabela 3

Correlação entre os Grupos de Critérios da CID-10 e as Variáveis do Rorschach para Esquizofrenia

\begin{tabular}{|c|c|c|}
\hline $\begin{array}{l}\text { Critérios } \\
\text { da CID-10 }\end{array}$ & $\begin{array}{l}\text { Variáveis do } \\
\text { Rorschach }\end{array}$ & $\begin{array}{r}\text { Coeficiente de } \\
\text { Correlação }\end{array}$ \\
\hline Grupo 1 & Perseveração & $0,705 * *$ \\
\hline Grupo 2 & $\begin{array}{l}\text { At (incluindo } R x \text { ) } \\
\text { Sexo } \\
M- \\
H d[\text { incluindo }(H d)] \\
\mathrm{H}+\mathrm{A}<H d+\mathrm{Ad} \\
\text { TipoVivência Introversivo }\end{array}$ & $\begin{array}{l}0,427 * * \\
0,480 * * \\
0,454 * * \\
0,508 * * \\
0,466 * * \\
0,404 * *\end{array}$ \\
\hline Grupo 3 & $\begin{array}{l}\text { Auto-referências } \\
\text { M none }\end{array}$ & $\begin{array}{l}0,622 * * \\
0,270 *\end{array}$ \\
\hline Grupo 4 & $(H)$ e $(H d)$ & $0,246 *$ \\
\hline Grupo 6 & $\begin{array}{l}\text { Neologismo } \\
\text { Negação } \\
\text { Respostas Desviantes (DR2) } \\
\text { Contaminação } \\
\text { Confabulação }\end{array}$ & $\begin{array}{l}0,748 * * \\
0,544 * * \\
0,389 * * \\
\mathbf{0 , 3 1 5 * *} \\
\mathbf{0 , 2 6 5 *}\end{array}$ \\
\hline Grupo 7 & $\begin{array}{l}\text { Extratensivo } \\
\text { Rejeição } \\
\text { Contaminação }\end{array}$ & $\begin{array}{r}0,237 * \\
0,415 * * \\
\mathbf{- 0 , 2 6 6} * *\end{array}$ \\
\hline Grupo 8 & $\begin{array}{l}\text { Coartação } \\
\text { (VIII+IX+X/R) } \downarrow \\
\sum C^{\prime} \\
\sum \text { Sombreados } \\
\text { Cor-Sombreado misto } \\
\text { MOR } \\
\text { Sentimento de Incapacidade }\end{array}$ & $\begin{array}{rl}0,228 & (*) \\
-0,245 & * \\
0,409 & * * \\
0,270 & * * \\
0,372 & * * \\
0,399 & * * \\
0,217 & *\end{array}$ \\
\hline
\end{tabular}

$* p<0,05 ; * * p<0,01$.
O grupo de critérios 2 da CID-10 - delírios de controle, influência ou passividade, claramente relacionados a movimentos do corpo ou membros, ou ações, sensações ou pensamentos específicos; percepção delirantes - está associado com as variáveis do Rorschach Conteúdos Anatômicos (At), Sexuais (Sex), Conteúdo de Detalhe Humano [Hd e $(H d)$ ], percepções de conteúdos vitais inteiros $(\mathrm{H}+\mathrm{A})$ serem menores do que as percepções desses conteúdos em detalhes $(H d+A d)$, Movimento Humano de Má Qualidade (M-) (ver Tabela 3).

Muitas vezes o paciente se sente vítima passiva de vozes imperativas ou de cenestesias estranhas. Essas cenestesias estranhas, impressões sensoriais internas do organismo, reiteradas vezes estão relacionadas com queixas tais como a de um dos pacientes da amostra do estudo "O capeta e sua legião do mal tentam dirigir os meus pensamentos... eles captam tudo o que eu penso desde quando eu nasci. As vezes eu acordo no meio da noite com essa legião me atacando". Em mulheres, essas queixas cenestésicas localizam-se principalmente nos órgãos genitais; reclamam de serem vítimas de relações sexuais, de defloramento e de masturbação (Paim, 1991). Tais fenômenos delirantes cenestésicos são frequentemente considerados persecutórios também e foram verbalizados por nove pacientes deste estudo durante a aplicação da técnica de Rorschach. Dentre essas queixas a mais comum foi "tem um homem que abusa de mim aqui no hospital todas as noites" sendo que algumas delas eram categóricas ao afirmarem que não tinha como vê-lo, às vezes por estar escuro, mas elas sabiam.

É bem provável que as preocupações com essas cenestesias se refletiram no Rorschach por meio do aumento das respostas relacionadas ao corpo como $A t$, Sex, $H d$ e $(H d) e$ pelo índice $H+A<H d+A d$. Muitas $A t$ sugerem exteriorização de angústias encobertas que manifestam pobreza da autoimagem e dificuldade para simbolizar a própria problemática, que passa a ser refletida em preocupações corporais do tipo hipocondríacas, como também indicam o uso da intelectualização para tolerar a ansiedade, a tensão e os sentimentos de frustração (Vaz, 1997). O aumento de Sex é comum quando as preocupações de ordem sexuais estão presentes. Esses conteúdos, quando descritos de modo rebuscado ou excêntrico, ou em formulações confabuladas ou contaminadas, são típicos de distúrbios esquizofrênicos (Rapapport, 1971). As $H d e(H d)$ muito frequentes revelam pobreza da integração da autoimagem. Finalmente, quando $H+A<H d+A d$ entende-se que a pessoa percebe com suscetibilidade o meio humano, tendo uma perspectiva distorcida de si mesma e do seu meio social e com uma tendência a apresentar marcados componentes paranóicos (Exner \& Sendín, 1999).

Exner e Sendín (1999) observaram que a análise da categoria de conteúdos humanos $[H,(H),(H d)$ e $H d]$ ajudam a precisar o diagnóstico de esquizofrenia, permitindo reforçar ou descartar a hipótese elaborada a partir 
do Índice de Esquizofrenia ${ }^{1}$ (SCZI). Segundo os autores esses conteúdos humanos seriam muito escassos ou ausentes, podendo ser acompanhados de fenômenos especiais ou, ainda, o $H$ ser igual a zero, prevalecendo, então, os outros tipos de conteúdos humanos $[H d,(H)$ e $(H d)]$. Essa configuração de conteúdos humanos aponta uma predisposição para relações interpessoais habitualmente muito empobrecidas. Além disso, Exner (2003) notou que respostas de anatomia e raio-x, acompanhadas de qualidade formal negativa $(F-)$ apareciam mais frequentemente nos protocolos de pacientes com esquizofrenia do que nos protocolos de não pacientes. Por sua vez, Exner, Murillo e Sternklar (1979, citado por Exner, 2003) identificaram essas respostas em pacientes com esquizofrenia que manifestavam delírios relativos ao seu próprio corpo.

A associação, feita pela CID-10, desses delírios aos movimentos do corpo ou membros, ou ações, sensações ou pensamentos especificos possivelmente se correlacionou no Rorschach com as projeções de $M$-. O $M$ denuncia fuga da realidade, o agir coagido, bloqueado, ansioso e impotente. Esse tipo de resposta no teste, provavelmente, está relacionado ao déficit de habilidades sociais, sendo mais comum ocorrer em pessoas ansiosas de relacionamento interpessoal pobre, receoso e tenso (Vaz, 1997; Weiner, 1966). O $M$ - também é sugestivo de processos de pensamento desviantes ou dissociados, especialmente quando em atitudes passivas. Segundo Exner (2003), essa codificação é muito rara em adultos não pacientes, observada em apenas $3 \%$ dessas pessoas, pouco comum nos protocolos de diferentes grupos de pacientes, variando entre 30 a $40 \%$, mas muito frequente em pacientes com transtorno esquizofrênico, cerca de $80 \%$ deles. $\mathrm{O}$ autor afirma que o $M$-, quando aparece duas ou mais vezes em um mesmo protocolo do teste, pode refletir pensamentos delirantes.

Finalmente, o Tipo de Vivência Introversivo no Rorschach demonstrou correlação com esses critérios da CID-10. Nesse contexto da esquizofrenia, a introversão relaciona-se, por um lado, com aquelas pessoas que se baseiam fortemente em suas próprias avaliações internas, ou seja, têm um compromisso com o modo ideacional de abordar as situações de solução de problemas e tomada de decisão. São pessoas que não se permitem tomar decisões sem antes ficar ruminando sobre suas consequências. O problema é que, no caso dos pacientes com esquizofrenia, as formas como pensam e refletem baseiam-se em interpretações equivocadas da realidade com muita frequência. Klopfer e Kelly (1952) e o próprio Rorschach (1921/1978) também encontraram esse tipo de vivência

\footnotetext{
${ }^{1}$ Esse índice desenvolvido por Exner (1999) foi revisado e substituído por uma medida de distúrbio do pensamento, o Perceptual-Thinking Index - PTI. O PTI não se trata de um índice de diagnóstico, mas de um indicador do tipo de dificuldade no que diz respeito ao pensamento e à precisão perceptiva, que podem ter um impacto persistente em toda a estrutura de personalidade da pessoa.
}

introversivo em pacientes com esquizofrenia do tipo paranóide.

Os dados da Tabela 3 apontam que as variáveis do Rorschach - Autorreferência e Movimento Humano Abstrato - correlacionaram-se com os critérios 3 da CID-10 - Vozes alucinatórias fazendo um comentário contínuo sobre o comportamento do paciente ou discutindo entre si ou outros tipos de vozes alucinatórias vindas de alguma parte do corpo.

Nesse sentido, o paciente crê que tudo se relaciona a ele, tanto as censuras quanto os elogios, sente-se o centro do mundo. No Rorschach, a variável que mais se aproxima dessas descrições é o fenômeno Autorreferência. A Autorreferência típica desses pacientes qualifica mais do que um egocentrismo ou narcisismo patológico, denota um transtorno profundo do juízo e do pensamento (Vaz, 1997). Observa-se nesse fenômeno a falha na distinção entre sujeito e objeto, a perda de contato com o mundo objetivo por dissociação do "eu".

Os distúrbios do pensamento que se assemelham a essas operações do tipo alucinatórias, de uma forma geral, foram observados por Exner (2003) nas respostas de Movimento Humano sem qualidade formal ( $M$ none). Essas respostas quase sempre são altamente simbólicas e centram-se em algum aspecto da emoção ou sensação por exemplo, quando alguns dos pacientes responderam que a mancha "parece a tristeza", "o vazio na alma", "a maldade que fazem com ele" ou "esse é o meu medo", desconsiderando o contorno da mancha, ou seja, ignorando o seu formato. Segundo o autor, os $M$ none estão relacionados com mecanismos de intelectualização, com preocupações pessoais que interferem na percepção adequada da realidade e com falta de controle ideativo que interfere na clareza do pensamento. Inclusive quando esta variável surge mais do que duas vezes em um mesmo protocolo Exner sugere que se trata de uma forma de distanciamento do tipo alucinatório. Essas respostas no teste também foram codificadas como Abstrações $(A B) \mathrm{e}$ foram descritas como comentários confabulatórios (Confabulação). Logo, a Abstração, no caso dos sintomas alucinatórios, provavelmente se desvelou junto com o código $M$ none (resposta codificada por Vaz (1997) como $F$ - e conteúdo abstrato).

A única variável do Rorschach associada a esse grupo 4 de sintomas da CID-10 - Delírios persistentes de outros tipos, culturalmente inapropriados e completamente impossiveis (Por exemplo: ser capaz de controlar o tempo ou entrar em comunicação com seres alienígenas) foi o Conteúdo Humano descaracterizado $[(H)$ e $(H d)]$, conforme os dados expostos na Tabela 3.

$\mathrm{O}$ aumento das respostas de $(H) e(H d)$ é comum em pessoas em que predominam os pensamentos mágicos, onipotentes, com problemas nas relações interpessoais. Pessoas com esse grupo de sintomas tendem a ser mais receosas, cautelosas e controladoras nos seus relacionamento, pois tendem a perceber as pessoas com capacidades sobre-humanas e imprevisíveis (Vaz, 1997). 
Nota-se que não houve qualquer correlação entre os critérios 5 da CID-10 e as variáveis do Rorschach. No entanto, cinco variáveis do Rorschach - Fenômeno de Neologismo, Negação e Respostas, Desviantes de nivel 2 (DR2), Contaminação e Confabulação - se mostraram correlacionadas aos critérios 6 da CID-10-Neologismos, paradas ou interpolação no curso do pensamento, resultando em incoerência ou fala irrelevante (ver Tabela 3). É importante salientar que todos os pacientes que revelaram este critério em seu diagnóstico de esquizofrenia, também manifestavam critérios de delírios típicos desse transtorno, ou seja, critério 2 ou 4.

$\mathrm{O}$ neologismo consiste em criar ou inventar novas palavras. No Rorschach, o Neologismo está relacionado com dificuldade de comunicação clara em função de sua característica bizarra e sincrética. Essa classificação também foi observada no Rorschach de esquizofrênicos por Resende (2001) e Sousa (1982).

As paradas ou interpolações no curso do pensamento, resultando em incoerência ou fala irrelevante, segundo Paim (1991), refletem imediatamente na linguagem, quando a pessoa inicia um assunto e subitamente se detém, interrompe a fala. Esses comportamentos parecem traduzir-se no Rorschach por uma descontinuidade do pensamento e a dificuldade de concentração, quer pela Negação de resposta ou pela presença do código especial $D R 2$. No caso específico do $D R 2$, o paciente verbaliza frases inadequadas ou irrelevantes no meio da resposta, constituindo uma clara divagação, como também verbalizações bizarras (Exner \& Sendín, 1999).

Outras duas variáveis do Rorschach, a Contaminação e a Confabulação, foram estatisticamente significativas nesse grupo de sintomas na CID-10 $(p<0,01$ e $p<0,05$ respectivamente), embora tenham ocorrido com frequências relativamente elevadas em muitos pacientes (ver Tabelas 4 e 5). Ambas revelam desvios cognitivos mais graves, sérias desorganizações do pensamento e pouco contato com a realidade, bem como indicam pensamento forçado, inadequado e absurdo (Vaz, 1997).

Os critérios 7 da CID-10 - Comportamento catatônico (excitação, postura inadequada ou flexibilidade cérea) negativismo, mutismo e estupor - estão fortemente correlacionados com as variáveis Tipo de Vivência Extratensivo e Rejeição no Rorschach. O Tipo de Vivência Extratensivo - típico de pessoas mais influenciáveis pelo ambiente, que diante de tensão externa têm facilidade para perder o controle emocional e apresentam motilidade excitável - foi apontado por Vaz (1997) como típico de pacientes com esquizofrenia com sintomas catatônicos.

$\mathrm{O}$ mutismo e o estupor - ausência de linguagem oral e de reação aos estímulos externos (Paim, 1991) - tornam inviável a aplicação da técnica de Rorschach, que exige a presença da fala. Nesse caso, o número elevado de Rejeições pode ser uma expressão desses sintomas, inviabilizando, em vários casos, a correção da técnica. Obser- vou-se durante a fase de coleta de dados que os pacientes que apresentavam esse grupo de sintomas, mesmo dispostos a participarem do estudo, não conseguiam responder à técnica. Ficavam olhando para o cartão por alguns minutos sem nenhuma menção de que responderiam ao estímulo. Quando muito, alguns deles davam respostas estereotipadas, por exemplo, "uma coisa estranha, que não sei o nome", preferencialmente nos cartões cromáticos (II, III, VIII, IX e X), uma vez que tendem a ser mais sintônicos ao que ocorre no ambiente.

Nesse sentido, os pacientes com esse grupo de sinais e sintomas emitiram poucas respostas durante a submissão ao Rorschach e a variável $R$ revelou-se estatisticamente significativa nesse grupo $(-0,224$, sig. 0,019$)$. A correlação negativa indica que pacientes com os menores números de respostas em seus protocolos tendem a ter esse grupo sintomas. Essa variável denuncia a pobreza de associações, de fantasias e ausência de elaboração de imagens.

As variáveis do Rorschach Coartação, Baixo Índice de afetividade, ¿C', $\sum$ Somb, Cor-Somb, MOR e SI revelaram-se altamente correlacionadas ao grupo de pacientes que apresentaram os critérios da CID-10 - sintomas "negativos": apatia marcante, pobreza de discurso e embotamento ou incongruência de respostas emocionais (Tabela 3).

$\mathrm{Na}$ apatia registra-se a falta de vivência de qualquer tipo de afeto - alegria, tristeza ou raiva. O indivíduo não se interessa por nada em sua vida. No embotamento há uma perda profunda de todo tipo de vivência afetiva observável e constatável pela postura do paciente (Dalgalarrondo, 2000). Tanto a apatia quanto o embotamento e a pobreza de discurso parecem estar correlacionadas no Rorschach pelo Tipo de Vivência Coartado. A Coartação, no Rorschach de pacientes com esquizofrenia, ratifica a paralisia afetiva $\left(\sum \mathrm{C}=0\right)$ e ideativa $(M=0)$, expressando a maneira tensa, rígida e fria de como eles lidam com os outros e com o mundo ao seu redor. Observa-se nesses pacientes o predomínio do distanciamento das ingerências de fantasias, desejos, pulsões e afetos. Esse fato demonstra o esforço de leitura objetivante do material, uma tentativa de agarrar-se de maneira extrema à realidade, impedindo assim qualquer deslocamento metafórico ou incidência delirante. Dessa forma, esses pacientes mantêm um equilíbrio precário, que é mantido devido a uma vida restrita e de esquiva: evita circunstâncias novas e desafiadoras que possam impor pressões psicológicas. Esse tipo de proteção é frágil e a estabilidade é precária. Quando as circunstâncias mudam repentinamente, essas pessoas sobrecarregam-se e estressam-se ou se desestruturam facilmente (Exner, 2003; Ilonen et al., 2004; Kaser-Boyd, 2006).

Outra variável no Rorschach que sugere esses sintomas de embotamento e incongruência de respostas emocionais é a Diminuição do número de Respostas nos Cartões VIII, IX e X (Índice de afetividade< 0,30). 
Essa variável é comum em pessoas que têm aversão a situações que envolvam expressões de sentimentos, pois se sentem constrangidas e, como consequência, há uma retração emocional que prejudica a adaptação interpessoal e social. Preferem não estar envolvidas em situações emocionalmente intensas (Exner, 2003; Ilonen et al., 2004; Kaser-Boyd, 2006; Vaz, 1997).

Esse grupo de sintomas da CID-10 manifestou-se de várias outras formas no teste: (a) com o aumento do $\sum S o m b$ e do $\sum C^{\prime}$, o que caracteriza a desolação afetiva profunda típica desses pacientes; (b) com o aumento de Cor-Somb, que revela ambivalência afetiva; sentimentos opostos, como amor e ódio, prazer e desprazer, expressos ao mesmo tempo, direcionados às pessoas e situações. Essa ambivalência afetiva marca uma tendência a se sentir confuso e inseguro quanto ao que sente. Prazer e dor associados ao mesmo evento, dificultando à pessoa discriminar seus sentimentos e limitando suas possibilidades de bem-estar. São pessoas que mesmo felizes sofrem com preocupações e tristezas (Exner, 2003); (c) Conteúdos Mórbidos (MOR): o aumento desses conteúdos, segundo Exner (2003), ocorre em pessoas com ideações pessimistas, que temem e desconfiam de toda ajuda e oportunidade. Acreditam que tudo continuará mal independente do que possam fazer. Essas respostas têm relação com as percepções internas de elementos disfóricos que a pessoa atribui aos objetos externos; (d) Aumento do SI: esse Sentimento de Incapacidade é observado com mais frequência em pessoas com transtornos afetivos (Vaz, 1997) e, provavelmente, em pessoas que apresentam cronicidade de seus sintomas no distúrbio esquizofrênico com predomínio dos sintomas negativos, que é o caso dos pacientes avaliados.

A Tabela 3 demonstra que as correlações entre a técnica de Rorschach e a CID-10 são possíveis, embora o Rorschach não seja um instrumento construído especificamente para aferição diagnóstica de acordo com os critérios da CID-10 (OMS, 1993), e por esse motivo, também não existe uma relação direta entre todos os critérios da CID-10 e as variáveis derivadas do Rorschach. Contudo essas correlações apontam que as variáveis do Rorschach, de fato, identificam e descrevem alguns padrões de comportamentos e experiências internas não adaptativas característicos dos critérios observáveis da CID-10 para o diagnóstico da esquizofrenia.

Para finalizar, a Tabela 4 expõe as correlações encontradas entre as quatro variáveis mais apontadas pelos autores estudados e os critérios da CID-10, e a Tabela 5 expõe a média e o desvio padrão dessas mesmas variáveis entre os participantes do estudo $(n=80)$, como também mostra quais seriam os valores normativos esperados na população brasileira de não-pacientes segundo Vaz $(1997,2006)$ e revela quantos pacientes apresentaram resultados semelhantes e diversos da população normativa em cada uma dessas quatro variáveis.

Tabela 4

Correlação entre os Critérios da CID-10 e as Variáveis do Rorschach para Esquizofrenia

\begin{tabular}{lrccc}
\hline Critérios da CID-10 & \multicolumn{4}{c}{ Variáveis do Rorschach } \\
\cline { 2 - 5 } & F-\% & Contam & Confab & Pop \\
\hline Grupo 1 & 0,180 & $-0,044$ & 0,061 & $-0,185$ \\
Grupo 2 & 0,121 & 0,170 & 0,027 & $-0,173$ \\
Grupo 3 & 0,202 & 0,174 & 0,102 & $-0,183$ \\
Grupo 4 & $-0,098$ & $-0,035$ & $-0,158$ & 0,153 \\
Grupo 5 & $-0,028$ & $-0,004$ & $-0,071$ & 0,106 \\
Grupo 6 & 0,182 & $\mathbf{0 , 3 1 5 * *}$ & $\mathbf{0 , 2 6 5 *}$ & $-0,200$ \\
Grupo 7 & 0,019 & $\mathbf{- 0 , 2 6 6 * *}$ & 0,023 & $-0,023$ \\
Grupo 8 & $-0,035$ & $-0,069$ & $-0,033$ & $-0,006$ \\
\hline
\end{tabular}

$* p<0,05 ; * * p<0,01$.

Tabela 5

Estatística Descritiva das Variáveis mais Características da Esquizofrenia na Técnica de Rorschach $(n=80)$ e os Valores Normativos Esperados para a População Brasileira

\begin{tabular}{lcccr}
\hline Rorschach & $M(D P)$ & $\begin{array}{c}\text { Valores } \\
\text { normativos } \\
\text { (Vaz, 2006) }\end{array}$ & $\begin{array}{c}\text { \% de sujeitos } \\
\text { dentro das normas } \\
\text { (Vaz, 2006) }\end{array}$ & $\begin{array}{r}\text { \% de sujeitos } \\
\text { fora das normas } \\
\text { (Vaz, 2006) }\end{array}$ \\
\hline F-\% & $52 \%(29,3)$ & $\leq 20 \%$ & $17,5 \%(n=14)$ & $82,5 \%(n=66)$ \\
Contaminação & $1,53(2,0)$ & 0 & $40 \%(n=32)$ & $60 \%(n=48)$ \\
Confabulação & $1,39(1,6)$ & 0 & $50 \%(n=40)$ & $50 \%(n=40)$ \\
Popular & $0,98(1,1)$ & $>5$ & $1,3 \%(n=1)$ & $98,7 \%(n=79)$ \\
\hline
\end{tabular}


Como pode ser observado nas Tabelas 4 e 5, a distribuição dessas variáveis entre os pacientes e a elevada frequência com que surgem nos protocolos, especialmente F-\% elevado e Pop baixo, as tornam características do paciente esquizofrênico independente do grupo de critérios da CID-10 F20. As variáveis F-\% elevado e Pop baixo no Rorschach, embora não sejam exclusivas dessa psicopatologia, revelaram-se, mais uma vez, como características de pacientes com esquizofrenia. Esse fato garante uma validade adicional a essas variáveis que têm sido confirmadas no diagnóstico dessa psicopatologia há várias décadas em diferentes culturas. As Contaminações e Confabulações mostram-se diretamente significativas para os pacientes que revelam em seu diagnóstico os critérios de número 6 , conforme foi discutido durante a exposição dos resultados.

\section{Considerações Finais}

De uma forma geral, este estudo buscou correlacionar os critérios da CID-10 e as variáveis do Rorschach no que tange ao diagnostico da esquizofrenia, explorando pontos de convergência entre os dois instrumentos. As correlações observadas indicam que há relações consistentes entre os mesmos, embora se tratem de instrumentos com referenciais diferentes no campo da psicopatologia: a técnica de Rorschach, um instrumento que descreve aspectos estruturais e dinâmicos da personalidade baseados em dados empíricos, e a CID-10, um instrumento de classificação categorial de síndromes clinicas qualitativamente diferentes e consistentes com o diagnóstico médico tradicional.

As correlações não foram muitas e também não foram altas. É bem provável que as poucas correlações estejam relacionadas às diferentes facetas da psicopatologia que foram avaliadas pelos dois instrumentos: deve-se considerar que os critérios da CID-10 são bastante estruturados e que neles se considera a observação direta de comportamentos observáveis, enquanto o Rorschach avalia aspectos que muitas vezes não podem ser observados diretamente, favorecendo a avaliação de aspectos psicodinâmicos da psicopatologia. De qualquer forma, a correlação entre os dois instrumentos pode enriquecer a compreensão das informações que cada um deles pode informar isoladamente. Além disso, pode-se inferir que a técnica de Rorschach pode explicar apenas uma pequena porcentagem de variância nos critérios da CID-10 para a esquizofrenia.

As principais correlações encontradas podem ser descritas de maneira sucinta da seguinte forma: o critério 1 da CID-10 relacionou-se neste estudo com automatismos mentais típicos de pessoas com distúrbios cognitivos e neurológicos, ou seja, com o fenômeno de Perseveração no Rorschach; o critério 2 estava associado às preocupações corporais exageradas, do tipo delirantes com marcados componentes paranóicos, que se destacam, especialmente, em pessoas mais introversivas, que tendem a imaginar e fantasiar muito mais do que agir e compartilhar seus afetos e emoções [At, Sex, $H d e(H d),(\mathrm{H}+\mathrm{A})$ $<(H d+A d), M-$ e Introversão no Rorschach]; o critério 3 correlacionou-se com as variáveis Autorreferencia e Mnone no Rorschach, indicando um transtorno profundo do juízo e do pensamento associado a um egocentrismo patológico, com preocupações pessoais que interferem na percepção adequada da realidade; o critério 4 correspondeu às variáveis $(H) e(H d)$ no Rorschach, que estão relacionadas às pessoas em que predominam os pensamentos mágicos, onipotentes, com problemas nas relações interpessoais; o critério 6 associou-se à variáveis do Rorschach típicas de transtornos cognitivos graves que se expressam por meio de verbalizações bizarras, divagações e descontinuidade do pensamento, bem como aos pensamentos forçados, inadequados e absurdos (Neologismo, Negação, DR2, Contaminação e Confabulação no Rorschach); o critério 7 foi o critério responsável por uma perda significativa de protocolos em função da ausência de respostas ao teste. Esteve especialmente relacionado ao estilo de vivência Extratensivo no Rorschach, próprio de pessoas mais influenciáveis pelo ambiente, que diante de tensão externa têm facilidade para perder o controle emocional e apresentam motilidade excitável; o critério 8 convergiu para uma série de variáveis no Rorschach que indicam dificuldades de lidar com questões no âmbito afetivo (Coartação, VIII+IX+X/R $\downarrow, \sum C^{\prime}$, $\sum$ Sombreado, Cor-Somb, MOR, SI).

O único critério da CID-10 que não esteve correlacionado com nenhuma das variáveis do Rorschach foi o critério 5 - Alucinações persistentes em qualquer modalidade, quando acompanhadas por delírios "superficiais" ou parciais, sem claro conteúdo afetivo, ou por idéias sobrevaloradas persistentes. Esse grupo de sinais e sintomas da CID-10 é considerado como um dos menos patognomônicos da esquizofrenia do que os sinais dos itens anteriores, que também dizem respeito a sintomas de alucinações e delírios. Além disso, esse grupo de sinais e sintomas foi o critério da CID-10 menos apontado pelos psiquiatras no diagnóstico de seus pacientes. É possível que a correlação entre este critério $5 \mathrm{e}$ as variáveis do Rorschach tenha sido prejudicada pelas questões apontadas anteriormente, como também pelo fato de todos os pacientes apresentarem mais de um grupo de sinais e sintomas da CID-10. Vários pacientes preenchiam, em média, três ou quatro critérios e alguns poucos revelavam até seis critérios para o diagnóstico da esquizofrenia. Este último aspecto pode tornar o estudo de correlação um pouco confuso.

É importante observar, também, que esse estudo apresentou algumas outras limitações. Os grupos de sinais e sintomas são bastante amplos, incluindo características diversas, como no próprio grupo 5. Esse aspecto também pode ser um obstáculo ao estudo correlacional, já que de um lado têm-se vários critérios para ser correlacionados com diversas variáveis do outro. Embora a quantidade de participantes tenha sido considerada adequada para a 
população de Goiânia, considerando a incidência de 1\% da população relativamente constante em todos os países, para um estudo correlacional entre esses dois instrumentos que trabalham com muitas variáveis, o ideal seria que os pacientes apresentassem um ou dois grupos de sinais e sintomas da CID-10, mas tal fato não foi condizente com os pacientes que se encontravam internados nos hospitais.

Além disso, não foi possível assegurar a confiabilidade da classificação dos oito critérios diagnósticos da CID-10 para a esquizofrenia, uma vez que apenas o médico, responsável pelo paciente, que definia quais eram os grupos de sintomas que se manifestavam. Assim, não foi realizado o coeficiente de concordância entre juízes na definição dos grupos de sintomas.

Diante dessas observações, sugere-se que novas pesquisas sejam realizadas e novos dados sejam acrescentados para suprir as deficiências apontadas e permitir o avanço do estabelecimento de convergências e divergências entre esses diferentes instrumentos que avaliam a esquizofrenia. Dessa forma, também se pode contribuir para o avanço do construto esquizofrenia em processos de avaliação dessa psicopatologia por meio de diferentes instrumentos que possuem esta finalidade de diagnóstico e descrição da doença.

De qualquer modo, as correlações mais salientes observadas neste estudo são logicamente plausíveis e teoricamente consistentes, o que é uma evidência de alguma validade convergente dos instrumentos utilizados. As correlações entre as variáveis dos dois instrumentos, de um modo geral, revelaram-se positivas, de baixas magnitudes e significativas. Os resultados encorajam a realização de novas investigações com uma amostra de paciente mais homogênea em relação aos grupos de sinais e sintomas do transtorno esquizofrênico detectado pelos critérios da CID-10.

As informações adquiridas por meio desse estudo correlacional poderão ser usadas em empreendimentos científicos de follow-up que visem ao uso das variáveis do Rorschach associadas aos critérios da CID-10 no diagnóstico da esquizofrenia para detectar com antecedência a predisposição para esse transtorno, uma vez que a técnica de Rorschach tem um alto valor preditivo de distúrbios psicopatológicos.

Outro aspecto que merece ser salientado é o fato de este estudo ter sido baseado no Sistema Klopfer de aplicação, correção e interpretação do Rorschach e, ao mesmo tempo, agregar variáveis do Sistema Compreensivo. Esta é uma prática que deve ser evitada, pois se corre o risco de utilizar variáveis que possuem pressupostos diferentes quando são codificadas nos diversos sistemas de Rorschach. Ressalta-se que, para este estudo, as variáveis selecionadas do segundo sistema não possuíam incompatibilidades com o sistema de correção de Rorschach adotado. Tratava-se de dados qualitativos que podem ser levantados nas verbalizações dos examinandos, durante a aplicação da técnica, sem entrar em choque com as duas formas de correção do Rorschach. Diga-se de passagem, Exner também se baseou em Klopfer para construir o seu sistema.

Concluindo, considera-se que este estudo, apesar de suas limitações, traz contribuições importantes para o diagnóstico da esquizofrenia por meio dos dois instrumentos utilizados. Muito mais do que isso, essa pesquisa aponta temas diversos a ser minuciosamente investigados posteriormente, especialmente no que diz respeito à perspectiva de convergência entre dois instrumentos teoricamente diversos que são utilizados para avaliar a mesma psicoptalogia.

\section{Referências}

Adrados, I. (1991). Teoria e prática do teste de Rorschach (11. ed.). Petrópolis, RJ: Vozes.

Adrados, I. (2004). A intuição do psicólogo: Técnicas de abordagem com uso do Rorschach. São Paulo, SP: Vetor.

Amparo, D. M. (2002). A simbolização na esquizofrenia: Um estudo fenomeno-estrutural com o método de Rorschach (Tese de Doutorado não-publicada). Universidade de Brasília, DF.

Barbetta, P. A. (2004). Estatística aplicada às Ciências Sociais. Florianópolis, SC: Editora da Universidade Federal de Santa Catarina.

Beizmann, C. (1968). El Rorschach em el niño de tres a diez años. Madrid, España: Aguilar.

Blatt, S. J., \& Wild, C. M. (1976). Schizophrenia: A development analysis. New York: Academic Press.

Bodoin, N. J., \& Pikunas, J. (1983). A Rorschach form-color percentage and reality testing in adult pactients and nonpatients. Journal of Personality Assessment, 47(6), 583-587.

Bohm, E. (1979). Manual del psicodiagnóstico de Rorschach (7. ed.). Madrid, España: Ediciones Morata.

Chabert, C. (1993). A psicopatologia no exame de Rorschach. São Paulo, SP: Casa do Psicólogo.

Colombo, M. A., Alonso, H. L, Barreira, R. B., Cordarini, M. S., Gravenhorst, M. C., Herrera, M. T., et al. (1993). El psicodiagnóstico de Rorschach: interpretación. Buenos Aires, Argentina: Klex.

Dalgalarrondo, P. (2000). Psicopatologia e semiologia dos transtornos mentais. Porto Alegre, RS: Artmed.

Exner, J. E., \& Sendín, C. (1999). Manual de interpretação do Rorschach para o Sistema Compreensivo. São Paulo, SP: Casa do Psicólogo.

Exner, J. E., Jr. (2003). The Rorschach: A comprehensive system. Vol. 1: Basic foundations and principles of interpretation $\left(4^{\text {th }}\right.$ ed.). Hoboken, NJ: Wiley.

Ilonen, T., Taiminen, T., Karlsson, H., Lauerma, H., Leinonen, K. M., Wallenius, E., et al. (2004). Neuropsychological subtyping of schizophrenia. Psychiatry Research, 129, 191199.

Jakobsen, K. D., Frederiksen, J. N., Hansen, T., Jansson, L. B., Parnas, J., \& Werge, T. (2005). Reliability of clinical ICD10 schizophrenia diagnoses. Nordic Journal of Psychiatry 59(3), 209-212. 
Kaser-Boyd, N. (2006). Rorschach assessment of paranoid personality disorder. In S. K. Huprich (Ed.), Rorschach assessment of the personality disorders (pp. 57-84). Mahwah, NJ: Lawrence Erlbaum.

Kleiger, J. H. (1999). Disordered thinking and the Rorschach. Hillsdale, NJ: Analytic Press.

Klinger, E., \& Roth, I. (1965). Diagnosis of schizophrenia by Rorschach patterns. Journal of Projective Techniques \& Personality Assessment, 29(3), 323-35.

Klopfer, B., \& Kelly, D. (1952). Tecnica del psicodiagnostico de Rorschach. Buenos Aires, Argentina: Paidos.

Koide, R., Chien, C. P., Iizuka, S., \& Morita, N. (2002). A mass of flesh: Schizophrenic Rorschach percepts. Comprehensive Psychiatry, 43(6), 474-477.

Nascimento, R. S. G. F. do (2007). Rorschach Comprehensive System data for a sample of 409 adult nonpatients from Brazil. Journal of Personality Assessment, 89(Suppl. 1), 35-41.

Organização Mundial de Saúde. (1993). Classificação dos transtornos mentais e de comportamento - CID-10. Porto Alegre, RS: Artes Médicas.

Paim, I. (1991). Curso de psicopatologia (11. ed.). São Paulo, SP: EPU.

Pasian, S. R. (2000). O psicodiagnóstico de Rorschach: Atlas, normas e reflexões. São Paulo, SP: Casa do Psicólogo.

Pasquali, L. (1999). Instrumentos psicológicos: Manual Prático de Elaboração. Brasília, DF: Laboratório de Pesquisa em Avaliação e Medida.

Passalacqua, M. A., \& Gravenhorst, M. C. (2005). Os fenômenos especiais no Rorschach. São Paulo, SP: Vetor.

Portuondo, J. A. (1972). El psicodiagnostico de Rorschach y la esquizofrenia. Madrid, España: Biblioteca Nueva.

Rapapport, D. (1971). Test de psicodiagnóstico psicológico. Buenos Aires, Argentina: Paidós.

Resende, A. C. (2001). Os processos primários, secundários e terciários no Rorschach: Uma compreensão fenomenológica e uma proposta de valoração. (Dissertação de Mestrado nãopublicada). Universidade Católica de Goiás, Goiânia, GO.

Rorschach, H. (1978). Psicodiagnóstico. São Paulo, SP: Mestre Jou. (Original work published 1921)

Santos, M. A. (1996). A representação de si e do outro na esquizofrenia: Um estudo através do exame de Rorschach. (Tese de Doutorado não-publicada). Instituto de Psicologia, Universidade de São Paulo, SP.

Shereshevski-Shere, E., Lasser, L. M., \& Gottsfeld, B. H. (1953). An evaluation for anatomy content and $\mathrm{F}+$ percentage in the Rorschach of alcoholics, schizophrenics and normals. Journal of Projective Techniques, 17(2), 229-233.

Silva, M. D. V. (1987). Rorschach: Uma abordagem psicanalitica. São Paulo, SP: EPU.

Sousa, C. C. de (1982). O método de Rorschach (3. ed.). São Paulo, SP: Editora da Universidade de São Paulo.

Traubenberg, N. R. (1998). A prática do Rorschach. São Paulo, SP: Vetor.

Vaz, C. E. (1997). O Rorschach: Teoria e desempenho (3. ed.). Porto Alegre, RS: Manole.

Vaz, C. E. (2006). O Rorschach: Teoria e desempenho II. São Paulo, SP: Casa do Psicólogo.

Volkow, N. D. (2009). Substance use disorders in schizophrenia - Clinical implications of comorbidity. Schizophrenia Bulletin, 35(3), 469-472.

Weiner, I. B. (1966). Psychodiagnosis in schizophrenia. New York: Wiley.
Wood, J. M., \& Lilienfeld, S. O. (1999). The Rorschach Inkblot Test: A case of overstatement? Assessment, 6, 341-349.

Wood, J. M., Lilienfeld, S. O., Garb, H. N., \& Nezworski, M. T. (2000). Limitations of the Rorschach as a diagnostic tool: A reply to Garfield (2000), Lerner (2000), and Weiner (2000). Journal of Clinical Psychology, 56, 441-448.
Recebido: 16/02/2010 $1^{a}$ revisão: 08/11/2010 $2^{a}$ revisão: $18 / 04 / 2011$ Aceite final: $12 / 05 / 2011$ 